\title{
Marlène Albert-Llorca
}

\section{Eaux thermales et eaux miraculeuses}

L'une des substances par lesquelles passent le pouvoir de guérison reconnu au site de Lourdes est, comme on le sait, l'eau de la source dont la Vierge, le 25 février 1858, aurait révélé la présence à Bernadette Soubirous. Conformément aux ordres de la Vierge, la voyante alla y boire et s'y laver le visage et, dès les jours suivants, si l'on en croit un des premiers chroniqueurs des apparitions, Antoine Clarens, on disait que cette eau avait «opéré (...) certaines cures. Aussi voit-on accourir chaque jour, avec des bouteilles, pour les remplir, un grand nombre de personnes ». L'auteur précise : "Que l'eau dont il s'agit possédât quelques propriétés curatives, il n'y aurait rien là d'étonnant. Nous croyons savoir qu'elle tient en dissolution une quantité considérable de calcium ou de chaux » (Laurentin, 1963, 4: 317). Dans les mois qui suivirent les apparitions, on procéda en effet à des analyses chimiques destinées à savoir s'il s'agissait d'une eau minérale, l'enjeu étant, bien entendu, de déterminer si les guérisons relevaient des lois naturelles ou du miracle. Ces analyses établirent que l'eau était des plus ordinaires (Laurentin, ibid. : 383).

Que la question des propriétés de la source de Massabielle ait été posée à une époque qui voit triompher le positivisme n'a rien d'étonnant : tous les débats du XIXe siècle sur les miracles de Lourdes s'inscrivent dans ce contexte intellectuel. Dans le chapitre "Le acque » de son livre sur Lourdes, Clara Gallini (1998) rappelle, à juste titre, que ce siècle est également l'âge d'or du thermalisme et que les Pyrénées sont une région où l'on trouve quantité de stations thermales : on en compte alors plus de cent, exploitant trois cent soixante-sept sourcesi. On comprend, dès lors, que la question eau médicinale / eau miraculeuse ait été précisément posée dans le cas de Lourdesii.

II reste que ce sanctuaire est loin d'être le seul où l'eau joue un rôle. C'est plutôt l'inverse qui est vrai ; sans être systématique, la présence d'une source miraculeuse dans un lieu saint est relativement fréquente. Or, lorsqu'on examine la zone pyrénéenne, française et espagnole, on constate que les sources "sacrées " sont rarement des sources d'eau minérale. Du moins en est-il ainsi lorsqu'il s'agit de sanctuaires mariaux, les seuls, à ma connaissance, à avoir fait l'objet d'inventaires précis. Je m'appuierai sur celui qu'établit, pour la Catalogne, le dominicain Narciso Camós au XVIle siècleiii : il y décrit cent trois sanctuaires consacrés à une image miraculeuse de la Vierge et les modalités de son culte et rapporte, chaque fois qu'il le peut, le récit de l'invention de l'image. Dans seize cas, celle-ci aurait été découverte dans ou à côté d'une source, dès lors dotée de vertus miraculeusesiv. Or, deux 
seulement sont des sources thermales ${ }^{\vee}$ : celle du sanctuaire de Caldes de Boí, où un berger aurait trouvé à la fois la statue miraculeuse et les sources qui y jaillissent ${ }^{\mathrm{vi}}$, et celle de la Virgen de Fuente Calda, près de Gandesa (Tarragone). Si l'on considère, à l'inverse, les sites thermaux des Hautes Pyrénées, on constate que peu d'entre eux sont aussi des lieux saints. Trois, certes, furent aménagés sous l'impulsion de membres du clergé ou exploités par une institution religieuse : Cauterets, dont les sources furent propriété de l'abbaye de Saint-Savin du Xe au XVIlle siècle, (Savès, 1984 : 56) ; Barèges, où un testament daté de 1396 comporte un legs à Sainte Marie-Madeleine du Bain, sans doute l'institution qui possédait ou gérait les thermes (Bove, ibid. : 35) ; Luz-Saint-Sauveur, qui doit son nom à l'existence, dans le quartier des bains, d'une chapelle dédiée au Saint Sauveurvii. En 1572, l'évêque de Tarbes fit construire à côté d'elle une station thermale, sur le fronton de laquelle il fit graver ce verset d'Isaïe : "Vos haurietis aquas de fontibus salvatoris » (Savès, ibid. : 71). Aucun de ces trois sites, à l'exception peut-être de LuzSaint-Sauveur, n'est investi d'une valeur religieuse, du moins depuis la fin du Moyen Ageviii.

C'est la question de cette disjonction entre eaux naturellement curatives et eaux miraculeuses que je voudrais examiner. Elle peut paraître, en effet, assez curieuse. Comme chacun le sait, les propriétés curatives de certaines eaux sont connues depuis l'Antiquité, voire même avant, et ce savoir n'est pas le propre des lettrés. Dans leur Mémoire sur les eaux minérales et les monuments thermaux des Pyrénées, publié en 1794, Lomet et Ramond écrivent de la source de Vizos, qui jaillit à $900 \mathrm{~m}$. d'altitude dans la vallée de Barèges: "Ces bains [qui eurent, précisons-le, une certaine renommée au XVle siècle] sont oubliés de tout le monde, excepté des habitants des lieux voisins qui ont conservé pour eux une grande vénération et en font un fréquent usage " (cité par Peyruc, ibid. : 89). Lomet et Ramond auraient pu dire à peu près la même chose de bien d'autres sources qui, n'ayant jamais été aménagées, n'étaient utilisées que par les habitants de leurs environs immédiats. Si donc on connaissait, bien avant le XIXe siècle, les propriétés médicinales de ces eaux, pourquoi ne pas avoir choisi d'installer des sanctuaires précisément dans les lieux où elles jaillissaient?

\section{La désacralisation chrétienne de la nature}

Si les eaux ont été utilisées à des fins curatives bien avant la naissance de la chimie et de la biologie, c'est que les moyens ordinaires d'observation permettent de savoir que certaines d'entre elles sont dotées de caractéristiques particulières. On peut difficilement ne pas s'apercevoir qu'une eau a une température plus élevée, voire beaucoup plus élevée que la normale. La chose a sans doute paru remarquable puisque les localités où jaillissent des sources d'eau chaude portent des noms dérivés du latin " calidum ». Mentionnons, pour la zone pyrénéenne, Cauterets, Eaux-Chaudes, 
Caldes de Boí, Les Escaldes, Caldetes etc. Lorsque les eaux minérales ne sont pas chaudes, elles se signalent à l'attention par leur odeur (les eaux sulfureuses sentent, parfois violemment, l'œuf pourri), leur goût (salé, piquant etc.) et leur aspect: couleur rougeâtre, présence de filaments mucilagineux etc. Il était aisé d'en déduire que ces eaux pouvaient avoir des effets particuliers sur l'organisme. Pas nécessairement bons, d'ailleurs: Bernard Palissy souligne au XVle siècle qu'elles peuvent être bénéfiques ou nuisibles, selon la nature des minéraux dont elles se chargent dans leur parcours souterrain (Palissy, 1580: 39). D'autres lettrés sont moins réservés. En 1659, un médecin de Bagnères-de-Bigorre, De la Guthère, classe les eaux thermales parmi les remèdes " composés " tout en précisant, comme B. Palissy, que les minéraux qui y sont contenus sont «les vrais principes de leur vertu » (1659: 4). C'est ce que pensaient déjà les naturalistes romains. Pline l'Ancien écrit ainsi des eaux thermales de Baïes, dans la baie de Naples : " les unes agissent par la vertu du soufre, d'autres par celles de l'alun, du sel, du nitre ou du bitume, quelques unes aussi par l'effet d'un mélange acide ou salé » (1972, L. XXXI, § 4-5).

Les effets des eaux thermales ou minérales, pour Pline et ses pairs, n'ont rien de "surnaturel ». II en allait sans doute autrement, à leur époque, pour bien des adeptes du thermalisme: les ex-votos retrouvés sur les sites thermaux témoignent que les guérisons consécutives aux cures étaient souvent attribuées à l'action de quelque divinité (Lafon, 2000 ; Bouet, 2003 : 297-99). Car, si l'Antiquité a formé l'idée de la physis et, en conséquence, d'une médecine "laïque " qui utilise, entre autres, les propriétés des eaux, elle n'a sans doute pas opéré avec la même rigueur que l'ont fait les lettrés de l'Europe moderne la distinction entre nature et surnature. Aussi, comme l'écrit Xavier Lafon, le prêtre côtoyait-il le médecin dans les stations thermales de l'Antiquité (2000: 21). Posant un Dieu unique et transcendant à la Nature, le christianisme pouvait plus difficilement s'accommoder de cette ambiguïté. Sans doute l'Eglise a-t-elle parfois, comme l'ont écrit les folkloristes (cf. par ex. Sébillot1968 : 216-19), christianisé des lieux, dotés ou non de sources d'eau minérale, où se déroulait le présumé "culte des eaux » ou, comme le suggèrent les exemples de Caldes de Boíix ou la Fuente Calda, identifié des eaux thermales à des eaux miraculeuses. II semble cependant que le christianisme ait distingué plus nettement que le monde antique le territoire du médecin - les sociétés chrétiennes ayant perpétué l'idée antique d'une médecine "laïque » - de celui du prêtre ou plus précisément du saint. Ce partage, s'agissant des eaux, me semble s'expliquer par deux facteurs qui ne sont pas, au demeurant, absolument séparables : les caractéristiques sensibles des eaux médicinales et leurs modes d'utilisation.

\section{Une odeur de soufre}


Les historiens situent l'essor ou, plutôt, la renaissance de la pratique thermale en Europe au XIlle siècle (Boisseuil, 2002 : 7). Comme l'a montré Gérard Gouiran (1985), Flamenca, un roman anonyme en langue d'oc du Xllle siècle, donne un témoignage assez précis sur les usages que les élites sociales de cette époque faisaient des eaux. Le texte raconte les amours illicites entre Flamenca, épouse d'Archambaut de Bourbon, et un jeune chevalier, Guillaume de Nevers. Follement épris de Flamenca, Guillaume va user d'un stratagème pour rencontrer sa belle à l'insu de son mari, extrêmement jaloux. Prétextant qu'il est malade et qu'il veut, en conséquence, "prendre les eaux », il loue une chambre dans un hôtel de Bourbon (aujourd'hui Bourbon-L'Archambault) appartenant au propriétaire d'une source thermale. L'hôtel jouxtant la pièce où se trouvent les bains, Guillaume fait creuser un tunnel qui relie sa chambre aux thermes. Flamenca utilise le même prétexte que lui pour obtenir de son époux la permission de se rendre aux bains " quatre fois la semaine ", l'auteur précisant avec humour qu'elle y va " plus volontiers qu'à l'église et devant les chapelles des saints » (v. 6004). D'août à novembre, les deux amants vont s'adonner tranquillement aux jeux de l'amour.

Selon Flamenca, Bourbon jouissait d'une certaine renommée : « II y avait beaucoup d'étrangers venus de France, de Bourgogne, de Flandre, de Champagne, de Normandie et de Bretagne, pour prendre les eaux » (v. 3795). Elles étaient en effet, si l'on en croit le texte, des plus efficaces: "Dans chaque établissement de bains, on trouvait un panneau sur lequel figuraient les maux pour lesquels il était efficace. N'y venait ni boiteux, ni éclopé qui ne s'en retournât complètement guéri s'il y restait le temps nécessaire » ( $v$. 1465-70). Célèbres, les bains de Bourbon n'étaient fréquentés que par des gens aisés : ils étaient privés et il fallait payer pour y accéder, sans doute assez cher puisque le texte précise : " on ne s'y baignait que si l'on était riche " (v. 1493). Ce statut variait selon les lieux. A la même époque, les thermes de Pouzzoles, en Italie, sont collectifs et gratuits; au XVe siècle, on trouve à Bade à la fois des bains privés et payants et deux bains à ciel ouvert accessibles à tous; à Digne, à la même époque, il semble que les thermes, gérés par la ville, étaient gratuits ou peu onéreux (Gouédo-Thomas, 1994: 14, Gouiran, 1985 : 175). Le stratagème utilisé par Guillaume dans Flamenca - un tunnel - suggère, par ailleurs, que l'endroit où les curistes prenaient les bains se trouvait sous l'auberge, et donc sous terre. II en allait en tout cas ainsi à Digne, où des documents d'archives datant de la même époque que le roman occitan parlent du bahn de la crota (le bain de la grotte) et dels bahns soteyrans (des bains souterrains). Enfin, au vu de certaines des miniatures illustrant les manuscrits du De Balneis Puteoloneis, écrit par Pierre d'Eboli entre 1212 et 1221 , on peut penser que les bains de Pouzzoles étaient accolés à la montagne qui les surplombe, voire installés dans une cavité couverte d'une coupole. 
Flamenca précise enfin les caractéristiques sensibles des eaux. Leur odeur : "les bains ont une odeur particulièrement forte et qui n'en a pas un besoin urgent n'y recourt pas volontiers » (v. 6750) et leur température : "Dans chacun des bains sourdait une eau si chaude qu'elle bouillait presque ; d'un autre côté, coulait une eau froide qui refroidissait la chaude » (v. 1475). Arrêtons-nous d'abord sur la température. Au Moyen Age et dans la période moderne, les curistes se soignent en buvant l'eau des sources, en prenant des douches, en se faisant administrer des lavements ou, dans certains endroits, des bains de vapeur ou de boue. Cependant, le bain est presque partout un élément essentiel de la cure, du moins lorsque la station dispose de sources d'eau chaude. Or, dans la logique ascétique du christianisme, le plaisir pris à prendre un bain chaud est suspect : il amollit et est supposé enflammer le désir sexuel${ }^{x}$. II faut ajouter à cela que certains sites thermaux, au Moyen Age, jouent un rôle assez analogue à celui des étuves (les bains publics à vocation hygiénique) qui étaient alors des lieux de plaisir, voire de prostitution (Leguay, 2002: 235). Dans les étuves comme dans les sites thermaux, hommes et femmes se dénudent, entièrement ou en partie, pour se baigner. Les bains sont souvent collectifs et, si ceux des hommes et des femmes sont généralement séparés, les uns et les autres peuvent se retrouver à loisir dans les lieux de repos qui y sont installés. Aussi un dicton suisse du XVle siècle affirme-t-il : "Que la femme veuille aller aux bains, et le mari n'est plus le maître » (cité par Leguay : ibid.). C'est bien la «leçon » qu'on peut tirer du Roman de Flamenca!

La température des eaux thermales n'aurait pas suffi, à elle seule, à les faire suspecter aux yeux de l'Eglise si leurs utilisateurs, tant dans le «paganisme » romain qu'au Moyen Age (et cela reste vrai à l'époque contemporaine), n'avaient associé finalités curatives et hédonisme. Selon Marilyn Nicoud, c'est même la recherche du plaisir corporel qui prime à l'époque médiévale, la médicalisation de la pratique thermale ne commençant qu'au XVe siècle: les médecins s'attachent alors à définir les propriétés thérapeutiques de chaque source et à imposer aux curistes les règles de leur «bon usage»(2002: 26-30). Plus suspecte encore dans la «logique du sensible » qu'a manipulée la " pensée sauvage » chrétienne, l'odeur des eaux médicinales: une odeur nauséabonde, du moins lorsqu'il s'agit d'eaux sulfureuses, comme le sont celles de Bourbon L'Archembault. En intitulant son article sur Flamenca "L'odeur de soufre des eaux thermales » et en soulignant en conclusion qu'autour " des établissements thermaux médiévaux s'élève une vapeur sulfureuse que l'on ne doit pas aux seules propriétés chimiques des eaux ", G. Gouiran rappelle avec humour que le soufre est marqué négativement dans la pensée chrétienne. Considérée dans l'ancienne chimie comme une condensation de la matière du feu, cette substance a été associée à l'enfer : celui-ci et tout ce qui en sort, la passion amoureuse notamment, sentent le soufre. Nous sommes bien aux antipodes de «l'odeur 
de sainteté ". Aussi négatif, enfin, l'aspect trouble des eaux thermales: le christianisme, comme bien des religions, valorise la pureté et, si les clercs retiennent surtout celle de l'âme, il n'en reste pas moins que son équivalent et son adjuvant - matériel ne peut être qu'une eau limpide ${ }^{x i}$.

C'est bien pourquoi la Vierge et les saints sont généralement associés à des sources d'eau claire et fraîche, de l'eau «normale » plutôt que de l'eau thermale ou fortement minéralisée. II est significatif, à cet égard, que démons et êtres maléfiques soient plutôt liés aux eaux dormantes et qu'il n'existe pratiquement pas, comme le souligne P. Sébillot, de légendes attribuant au diable le jaillissement d'une source (1968: 186). Il reste que le même Sébillot a recueilli un ensemble de récits de tradition orale sur la découverte des sources thermales qui conduit, sinon à nuancer la distinction entre eaux miraculeuses et médicinales, du moins à préciser la place de ces dernières dans le monde naturel.

\section{Le miraculeux et le merveilleux}

Je me suis surtout appuyée jusqu'ici sur des écrits qui renseignent assez précisément sur les usages que faisaient les élites sociales des eaux thermales, mais beaucoup moins sur leurs utilisations populaires. Les collectes des folkloristes ne sont guère plus riches : intéressés par tout ce qui relevait, à leurs yeux, de la "superstition » et de la " magie ", ils ont beaucoup parlé des "fontaines sacrées » et beaucoup moins des eaux médicinales. On sait pourtant, j'y ai déjà fait allusion, que les cures par les eaux ne se déroulaient pas seulement à l'intérieur des établissements thermaux. Comme le révèlent, pour les Pyrénées, le Mémoire de Lomet et Ramond et, dans d'autres régions, des enquêtes du même typexii, bien des sources thermales qui étaient au XVIlle siècle à leur état natif étaient connues et utilisées par les gens du lieu. Certaines d'entre elles, dans les Pyrénées, furent aménagées et exploitées au cours du XIXe siècle, parfois de façon éphémère, mais d'autres ne l'ont jamais été, étant isolées dans la haute montagne et donc difficilement accessibles (certaines se trouvent à des altitudes supérieures à $1500 \mathrm{~m}$.) ou ayant un débit trop faible pour qu'il soit rentable d'amener leur eau jusqu'à une station thermale.

Si l'on sait que les eaux thermales étaient utilisées localement, on ne sait guère comment elles l'étaient précisément. Force est donc de se fonder, dans ce domaine, sur les rares données dont on dispose pour le passé et les enquêtes effectuées dans le présent, certaines sources étant encore utilisées aujourd'hui de façon coutumière. Parmi ces enquêtes, celle de Federica Tamarozzi en Corse. Elle y relève que la "sorgente termale [non è mai] investito dei poteri del sacro" (1999: 47). Mes incursions dans la zone pyrénéenne vont aussi dans ce sens.

On peut noter, ainsi, qu'aucun signe religieux ne figure sur les fontaines d'eau médicinale alors qu'on a très souvent dressé une statuette d'un saint 
ou de la Vierge sur les fontaines d'eau «normale "xiii. Egalement significative, l'étendue du champ d'utilisation des eaux thermales. Employées pour soigner les hommes et surtout, semble-t-il, les bêtes malades, elles l'ont aussi été à des fins non curatives. Joan Amades signale que les fabricants de paniers de Caldes de Montbui se servaient des eaux du lieu pour ramollir les tiges de jonc destinées à être tressées (1956, I: 539). Les bassins où se déversent les eaux chaudes ont souvent, par ailleurs, servi de lavoirs. Toutes les eaux chaudes, certes, ne sont pas médicinales ou ne sont pas considérées comme telles. Ainsi, aux abords de l'agglomération de Bagnères-de-Bigorre se trouve un établissement thermal, aujourd'hui fermé, qu'alimentait une source d'eau chaude et sulfurée, la source du Salut. A une centaine de mètres de cet établissement jaillit une source d'eau tiède (en réalité, une résurgence) qui se déverse dans un bassin grossièrement aménagé : sur un bord, on a placé de grandes pierres plates sur lesquelles les femmes venaient autrefois frotter leur linge ; ce bassin ne sert plus de lavoir, mais ses eaux continuent d'être utilisées par les habitants de la ville et des environs, pendant l'hiver, pour laver les boyaux du cochon qu'ils viennent de tuer.

$\mathrm{Si}$ les Bagnérais ont nettement distingué les eaux chaudes des eaux thermales, il est aussi des endroits où ces dernières ont été utilisées à la fois à des fins thérapeutiques et hygiéniques : au Moyen-Age, les deux bains de Bade qui étaient ouverts à tous servaient aussi de lavoir (Gouédou-Thomas, 1994 : 13) ; à Guitera, en Corse, les femmes viennent étendre les pièces délicates de leur linge dans le bassin public qui reçoit les eaux thermales de la localité ; toujours en Corse, certains vont se laver dans des sources thermales après une journée de travail ou de chasse (Tamarozzi, op. cit. : 51, 48, 50). Laver son linge dans une eau sacrée est considéré comme une profanation ${ }^{\mathrm{xiv}}$. Qu'on s'autorise à le faire dans les sources thermales suggère qu'on les distingue bien des eaux miraculeuses.

Vont également en ce sens les légendes narrant la découverte des eaux thermales recueillies par $P$. Sébillot. Beaucoup moins nombreuses que celles qui concernent les sources "sacrées ", elles s'en distinguent en ceci qu'elles ne racontent pas la naissance de la source mais la façon dont on l'aurait découverte (1968, II : 188-89). Le médiateur est toujours un animal : une chèvre, un mouton ou une vache malade qui s'en allait boire chaque jour à la source; un cheval qui guérit après s'être plongé dans l'eau ; un sanglier que l'on retrouva intact, plusieurs jours après sa mort, parce qu'il était allé s'échouer dans un ruisseau d'eau saline. Comme le souligne P. Sébillot (op. cit.: 188), la découverte ne se produit presque jamais dans des " circonstances miraculeuses ", ni même « merveilleuses " ${ }^{\times v}$.

Il en va de même en Corse: "Non una, fra le numerose raccolte dei raconti e del materiale fantastico locale fa diretta menzione delle acque termali. [Nessun raconto da] alcuna informazione sulla loro origine. (...) le sorgente termali vengono considerate figlie del caso naturale 0 , comunque, 
facenti parte di una creazione della quale sarebbe sciocco chiedere ragione » (Tamarozzi, op. cit. : 51). Pas de récits d'origine, donc, mais deux légendes sur leur découverte très proches de celles que mentionne P. Sébillot: à Puzzichello, la découverte des vertus des sources d'eau sulfureuse froide serait due à un troupeau de chèvres affecté par une épidémie de fièvre aphteuse (afta epizootica) ; les sources d'eau chaude de Guitera auraient été trouvées par des femmes qui allaient rincer leur linge à la rivière. Le second récit suggère une trouvaille due au hasard. Le premier, comme ceux qu'a collectés P. Sébillot, est plus ambigu: dire qu'un animal a découvert une source thérapeutique, est-ce dire qu'il y a là un fait de hasard ou l'effet d'une volonté providentielle?

La question se pose dès lors qu'on rapproche ces récits des légendes sur l'invention des images miraculeuses de la Vierge. Ici encore, c'est toujours un animal (un taureau, une brebis etc.) qui permet à son berger de découvrir l'image : voyant sa bête quitter le troupeau chaque jour, il la suit et trouve la statue sur un arbre, près d'une source etc. Dans ces légendes, on sait que l'on se trouve face à un événement miraculeux parce qu'on a affaire à une image religieuse, qui manifeste immédiatement ses pouvoirs surnaturels: transportée à l'église paroissiale, elle revient d'elle-même à l'endroit où elle a été découverte. Rien de tel dans les récits sur les sources thermales. Comment expliquer alors le rôle qu'ils donnent à l'animal ?

Cela peut tenir au fait que ces eaux ont été utilisées pour soigner les animaux aussi bien que les humains. Certaines, d'ailleurs, le sont encore. Ainsi, en toute saison (comme j'ai pu le constater, m'y étant rendue en février, après de fortes chutes de neige), les habitants de Bagnères-de-Bigorre et des villages environnants vont à la fontaine de Crastes, qui coule à quelques centaines de mètres au-dessus du village d'Asté et est réputée soigner les eczémas et les ulcères variqueux : ils y puisent de l'eau qu'ils emportent pour la boire ou en faire des lotions. A également été construit un bassin plus particulièrement utilisé, bien qu'un panneau l'interdise, pour baigner les animaux affectés d'une maladie de peauxvi. Soulignons qu'il se trouve un peu en contrebas de la fontaine, cela évitant que l'eau destinée aux humains ne soit souillée. Dans bien d'autres cas, les sources ont servi surtout, voire uniquement, à soigner les animaux. C'était le cas, il y a quelques années, de la fontaine du Banhet, dans la vallée de Campan, où l'on amenait plus particulièrement les chiens atteints de maladies de peau. Une légende, recueillie par Xavier Ravier dans le hameau de Gripp, qui se trouve à quelques centaines de mètres de la source, assure que ses propriétés furent découvertes parce qu'un mouton y allait boire (1986:83-85). II en allait de même de la source de Labarthe-de-Neste, appelée "Hount des cas" (Fontaine des chiens) parce qu'elle avait la réputation de guérir les chiens atteints de la gale (Peyruc, $1984: 100$ ). 
Cependant, pour comprendre pourquoi l'on a attribué à des animaux la découverte des vertus des eaux médicinales, il faut aussi tenir compte des représentations qu'on se fait de l'animal. Dans nos sociétés, comme l'attestent aussi bien les sources «savantes » que les données de la tradition "populaire », il a été considéré à la fois comme infra-humain et comme possédant des savoirs et des pouvoirs supérieurs à ceux des hommes. Que l'on songe, par exemple, aux innombrables récits ou proverbes prêtant aux animaux la capacité de prévoir le temps, d'annoncer la mort etc. C'est dans cet horizon qu'il faut situer les récits qui leur prêtent la découverte des eaux médicinales.

Cela signifie-t-il que les vertus de ces eaux, à défaut de se situer dans le " miraculeux », s'inscrivent du moins dans le champ des phénomènes désignés, à l'époque médiévale et au début de la période moderne, comme « merveilleux »? Ce terme, comme le relève Marilyn Nicoud (2002 : 21-22), est fréquemment employé par les médecins italiens du XIVe-XVe siècles pour qualifier les eaux thermales: elles ont "des effets merveilleux », entendons qu'elles sont capables de guérir toutes les maladies (comme le disait aussi, on s'en souvient, l'auteur de Flamenca)xvii. Encore faut-il souligner que la catégorie du merveilleux n'est pas dénuée d'ambiguïté : elle peut qualifier des réalités étonnantes, mais relevant de la nature, référer à un surnaturel non chrétien (les fées et autres entités du même genre), ressortir à la magie, voire être utilisée comme un synonyme de miraculeux (Nicoud, $2002: 21$, Le Goff, 1978)xviii. Pour déterminer ce qu'il en est dans les conceptions communes de notre époque, il faut préciser quels effets on prête à ces eaux et de quelle manière on en use.

\section{Façons de faire}

F. Tamarozzi a recueilli, en Corse, plusieurs récits, parfois racontés à la première personne, concernant des malades qui eurent recours aux eaux thermales, leur cas ayant été jugé désespéré par les médecins ${ }^{\text {xix }}$ : un homme atteint d'un cancer, un autre quasiment paralysé par ses rhumatismes, une enfant affectée par un eczéma qui avait résisté à toutes les médications etc. La guérison serait intervenue à des vitesses variables: le rhumatisant fut soulagé dès son premier bain, le cancéreux se baigna deux fois par jour durant une longue période, l'eczéma disparut au bout d'une cure de vingt-et-un jours (op. cit. : 51). Un homme, rencontré à la fontaine de Crastes, se montra plus réservé. Craignant d'avoir un ulcère à l'estomac, il faisait régulièrement « des cures de huit jours » avec l'eau qu'il venait chercher; sa femme, quant à elle, l'avait employée en bains de siège pour ses hémorroïdes - qu'il avait fallu néanmoins, précisa-t-il, soigner chirurgicalement. Rien d'étonnant dans cette diversité d'attitudes. Ce type de cures n'étant pas réglé par le corps médical (ou les " guérisseurs populaires ", qui édictent aussi des règles), chacun doit s'en remettre à son jugement, ce que fit aussi Montaigne, peu convaincu de la 
validité du savoir médical de son temps, dans son parcours thermal. II reste que presque tous les cas cités font apparaître que les façons "populaires » d'utiliser les eaux ont subi l'influence des normes posées par le corps médical dans les stations thermales: la cure doit avoir une certaine durée, il faut prendre un bain par jour, boire plusieurs fois dans la journée etc.

Le modèle médical a également eu une influence sur Lourdes: " $E$ proprio il modello curativo del termalisme ottocentesco a fare adottare (...) una bipartizione che doveva figurare come necessaria, adeguata allo stile dei tempi nuovi. Da un lato, il bere alle fontanelle, con il relativo spazio per la socializzazione e gli incontri. Dall'altro, l'immersione nelle vasche d'acqua, come pratica individuale e separata » (Gallini, 1998 : 104). Par ailleurs, alors même que le miracle est supposé instantané, il arrive que les pèlerins utilisent l'eau de façon répétée, comme on le fait dans les stations thermales. Certains vont se baigner chaque jour dans les piscines durant leur pèlerinage; presque tous vont boire plusieurs fois dans la journée aux fontaines. Et n'est-ce pas l'interdit, posé dans les cures thermales, de se sécher après le bain de façon à laisser agir les minéraux le plus longtemps possible qui a suscité la croyance commune qui veut qu'on ressorte sec des piscines de Lourdes? Piscines, certes, qui prenaient au XIXe siècle, comme les eaux thermales malodorantes ou troubles, le contre-pied de l'exigence de limpidité des eaux miraculeuses puisqu'y flottaient, comme le racontent Zola et Huysmans, les traces purulentes du passage de certains malades...

Ces discours et ces pratiques suggèrent que la frontière qui sépare les eaux thermales des eaux miraculeuses n'est pas dénuée d'une certaine porosité. II me semble néanmoins difficile de conclure de la similitude de certains usages à une confusion des genres. Je crois avoir donné quelques raisons de penser que le "peuple», pas plus que les lettrés, n'a confondu eaux miraculeuses et eaux "naturellement » médicinales. II reste à cerner les représentations de la «nature » et de ses pouvoirs qui fondent, pour nos contemporains, la croyance dans les vertus des eaux thermales. Elles ne sont certainement pas homogènes, pas plus que le sont les usages. En proposant, avec une parfaite conscience de faire un anachronisme, de reprendre la notion médiévale de "merveilleux » qu'ont commencé à explorer les historiens de cette période, j'ai simplement voulu pointer la difficulté, à défaut de la résoudre.

${ }^{\text {i }}$ L'essor de Lourdes, au demeurant, n'est pas sans rapport avec le succès du thermalisme puisque la décision d'ouvrir une «route thermale» permettant de relier les stations 
pyrénéennes est prise par Napoléon III en 1859, un an après les apparitions (Desplans, 1989 : 17).

${ }^{i i}$ La question, cependant, a été soulevée avant le XIXe siècle. En 1725, Dom Lobineau écrit dans $L a$ vie des saints de Bretagne, à propos des vertus prêtées à l'eau de l'île de Saint Maudet: "Comme on rapporte la même merveille de plusieurs autres saints (...), il se pourrait bien, supposé que le fait soit vrai, que cette guérison ne devrait s'attribuer qu'à la salure et à l'amertume de l'eau de mer dont la terre de ces isles est imbibée, ou à quelque autre cause naturelle dont nous laissons la découverte aux philosophes » (cité par Prigent, $1994: 31)$.

iii La première édition de l'ouvrage date de 1657. J'ai utilisé une édition du XVIIIe siècle. Sur cet ouvrage et le légendaire dont il fait état, voir Christian, 1981 et Albert-Llorca, 2002.

iv Camós ne dit pas, cependant, de toutes les sources qu'elles ont un usage thérapeutique : certaines étaient utilisées, selon lui, en cas de sécheresse : on y transportait la statue pour faire venir la pluie.

${ }^{\vee}$ En dehors des monographies détaillées qu'il a établies, Camós donne aussi, pour chaque diocèse catalan, le nom et la localisation des autres chapelles et églises dédiés à la Vierge Parmi eux, celui de la Virgen de Gracia à Villeneuve Les Escaldes (Cerdagne), qui possède aussi des thermes.

${ }^{v i}$ Caldes de Boí est aujourd'hui une station thermale très réputée en Catalogne : elle compte 37 sources, chaudes et froides.

vii L'existence de la chapelle est attestée depuis 1495.

viii Le cas de Luz Saint-Sauveur mériterait une enquête plus précise. Aux faits déjà mentionnés, il faut ajouter une légende attribuant la découverte des vertus des eaux (supposées agir sur les fonctions reproductrices) à une "dame avec de grandes ailes » qui serait apparue pour conseiller à une femme stérile de se baigner dans la source ; neuf mois plus tard, celle-ci donnait naissance à des jumeaux. Ange ? Fée des eaux ? L. Saves, qui rapporte ce récit, n'en dit pas plus et ne précise pas non plus quand ni comment il l'a collecté.

${ }^{\text {ix }}$ Soulignons que, à Caldes de Boí, aucun document ou vestige archéologique ne permet de penser qu'il existait des thermes à l'époque romaine.

${ }^{x}$ Les bains froids, à l'inverse, sont un antidote au désir charnel. Dans la littérature médiévale, les bains froids ou, à l'inverse, bouillants sont aussi la punition infligée aux femmes luxurieuses (Clier-Colombani, $1991:$ 171-75).

${ }^{x i}$ Un contre-exemple, la source du sanctuaire de la Vierge de Montgrony, en Catalogne, utilisée pour ses propriétés lactifères. L'eau « dépose sur les parois [de la fontaine] une chose blanche, qui ressemble à du lait et a la consistance du bitume. Les fidèles en prennent par dévotion et sont grandement soulagés de leurs maux lorsqu'ils l'appliquent sur leur corps » (Camós op. cit. : 302).

${ }_{\text {xii }} \mathrm{Au}$ XVIIIe siècle, les pouvoirs publics font en effet effectuer des enquêtes sur les ressources locales qui intègrent l'examen des sources. Ainsi, le Mémoire historique et statistique du département de la Lozère publié à Mende en l'an X, sous la direction du premier préfet du département. Il dit de la source thermale de La Chaldette: «il n'y a ni bassin, ni établissement public. Elle ne sert qu'aux habitants du voisinage » (Bardy 1994 : 213).

xiii Je dois cette remarque à Béatrice Morisson qui m'a aussi guidée à Bagnères-de-Bigorre. Je l'en remercie et remercie aussi Raphä̈le Garreta et Sébastien Lay.

${ }^{\text {xiv }}$ Pour des exemples de profanation d'une source sacrée et des châtiments qui s'ensuivent, voir Camós (op.cit.: 137, 300- 303) sur la Vierge de La Fuente de la Salud et celle de Montgrony . Le premier cas est commenté par Christian, op. cit. : 173-80.

${ }^{\mathrm{xv}}$ Font exception le récit sur Luz-Saint Sauveur que j'ai cité à la note 7 (et que Sébillot ne mentionne pas) et une légende sur la découverte des eaux de Bagnères-de-Bigorre recueillie à 
la fois par E. Cordier et J.-F. Bladé. L'agent est aussi un animal, vache ou taureau, mais, comme le souligne Sébillot, ces récits inscrivent l'événement dans «des conditions plus merveilleuses » (op . cit. : 189). La découverte des sources est en effet insérée dans un vaste récit d'origine qui a pour but d'expliquer le mode d'occupation actuel de l'espace montagnard (alpages dans les hauteurs et habitat et cultures dans les vallées, les eaux thermales se situant entre les deux). Le récit fait remonter ce système écologique à l'avènement du christianisme, lui-même contemporain de la première chute de neige. Il décrit donc la fin d'une sorte d'âge d'or et c'est sans doute pour cette raison qu'il intègre la découverte des sources thermales. Bien des mythologies expliquent en effet que les maladies n'existaient pas dans le temps des origines. Sur ces récits, cf. Ravier, 1986.

${ }^{\text {xvi }}$ Sur ce type d'usages en Corse, voir Tamarozzi, op . cit. : 49.

xvii Un dicton affirme aussi : «Cauterets, que tot guaritz»(Cauterets guérit de tout). Les curistes du XIIIe siècle pensaient certainement, en outre, que les eaux produisaient des guérisons instantanées puisque Flamenca précise, comme on l'a vu : «N'y venait ni boîteux, ni éclopé qui ne s'en retournait complètement guéri s'il y restait le temps nécessaire " (soul. par moi). Au XVIIe siècle, De la Guthère (1659) prend également soin de préciser : " on se doit instruire à ne pas atteindre une santé parfaite et subite en sortant des eaux » .

xviii Nicoud (op. cit.: 21-22) souligne en effet que le partage eaux naturellement / miraculeusement thérapeutiques n'est pas toujours très clair au Moyen Age.

xix $\mathrm{Au}$ XVIe siècle, c'est aussi en ces termes que Marguerite de Navarre évoquait, dans le prologue de son Heptaméron, les effets des eaux de Cauterets: " [Ce] sont choses si merveilleuses que les malades abandonnez des médecins s'en retournent tous guariz ».

\section{Bibliographie}

ALBERT-LLORCA, Marlène, 2002. Les Vierges miraculeuses. Légendes et rituels. Paris, Gallimard, coll. « Le temps des images ».

AMADES, Joan, 1956. Costumari Català. El curs del any, Barcelona, Salvat Editores, 5 vol.

BARDY, Bernard, 1994. «Vogue et destin d'une station thermale de montagne, La Chaldette ", in Villes d'eaux. Histoire du thermalisme.Actes du $117^{e}$ congrès national des sociétés savantes. Paris, Eds du CTHS.

BOISSEUIL, Didier, 2002. «Espaces et pratiques du bain au Moyen Age », Médiévales, « Le bain : espaces et pratiques», D. Boisseuil (coord.), Presses Universitaires de Vincennes, n. $43: 5-11$.

BOUET, Alain, 2003. Les thermes privés et publics en Gaule Narbonnaise.I. Synthèse. Collection de l'Ecole Française de Rome.

BOVE, Jean-Pierre, 1984. «Barèges, la plus haute station de France », », in Le thermalisme dans les Hautes-Pyrénées. Catalogue d'exposition, Lourdes, Musée Pyrénéen.

CAMÓS, Francisco, s. d. Jardin de Maria plantado en el principado de Cataluña, Gerona, Joseph Bró impr.(1 $1^{\text {ère }}$ ed. : 1657, Barcelona, Jayme Plantada). 
CHRISTIAN, William A., 1981. Apparitions in Late Medieval and Renaissance Spain. Princeton, Princeton University Press.

CLIER-COLOMBANI, Françoise, 1991. La fée Mélusine au Moyen Age. Images, mythes et symboles. Paris, Le Léopard d'Or.

DE LA GUTHERE, 1659. Du bon usage des eaux de Baignères, A Tolose, chez I. Dominique Camusat, Marchand Libraire au Palais.

DESPLAN, Marylise, 1989. Le thermalisme haut-pyrénéen au XIXe siècle. Thèse de médecine (dact.)

GALLINI, Clara. 1998. Il miracolo e la sua prova. Un etnologo a Lourdes. Napoli, Liguori Editore.

GOUEDO-THOMAS, Catherine, 1994. «Le thermalisme médiéval, de Flamenca à Michel de Montaigne, récits et images » in Villes d'eaux. Histoire du thermalisme.Actes du $117^{e}$ congrès national des sociétés savantes. Paris, Eds du CTHS.

GOUIRAN, Gérard, 1985. «L'odeur de soufre des eaux thermales. A propos de Flamenca et de textes médiévaux non littéraires », Sénéfiance, «L'eau au Moyen Age », n. 15 : 173-184.

LAFON, Xavier, 2000. «Thermalisme et mythologie. Le prêtre et le médecin », in M. Moldoveanu : Cités thermales en Europe, Actes Sud : 11-22.

LAURENTIN, René. 1961-1964. Lourdes. Histoire authentique des apparitions. LourdesRome, P. Lethielleux Editeur, 6 vol.

LE GOFF, Jacques, 1978. «Le merveilleux dans l'Occident médiéval », in L'étrange et le merveilleux dans l'islam médiéval. Actes du colloque tenu au Collège de France à Paris, mars 1974. Paris, 1978 : 61-80.

LEGUAY, Jean-Pierre, 2002. L'eau dans la ville au Moyen Age, Rennes, Presses Universitaires de Rennes.

MARGUERITE dANGOULEME, reine de NAVARRE 1492-1549, 1999. Heptameron. Ed. critique de R. Salminen, Genève-Droz

MONTAIGNE, Michel de, 1967. Journal de voyage en Italie, par la Suisse et l'Allemagne en 1580 et 1581, in Euvres complètes, Paris, Eds du Seuil.

NICOUD, Marylin, 2002. «Les médecins italiens et le bains thermal au Moyen Age », Médiévales, «Le bain : espaces et pratiques», D. Boisseuil (coord.), Presses Universitaires de Vincennes, n. $43: 13-40$.

PALISSY, Bernard, 1580. Discours admirable de la nature des eaux et des fontaines, tant naturelles qu'artificielles, chez Martin Le Jeune, in CEuvres complètes, Ed. sous la dir. De Marie-Madelaine Fragonard, Mont-de-Marsan, Eds Interuniversitaires, T. II. 
PEYRUC, Georges, 1984. «»; «Stations et sources oubliées ou abandonnées » in Le thermalisme dans les Hautes-Pyrénées. Catalogue d'exposition, Lourdes, Musée Pyrénéen.

PLINE L'ANCIEN, 1972. Histoire Naturelle, Livre XXXI, texte établi, traduit et commenté par Guy Serbat, Paris, « Les Belles Lettres ».

PRIGENT, Christiane. 1994. "Les fontaines thérapeutiques en Bretagne », in Villes d'eaux. Histoire du thermalisme.Actes du $117^{e}$ congrès national des sociétés savantes. Paris, Eds du CTHS.

RAVIER, Xavier, 1986. Le récit mythologique en Haute-Bigorre, Aix-en-Provence, Edisud / Eds du CNRS.

Flamenca : roman occitan du XIIIe siècle, 1988. Texte établi, traduit et présenté par JeanCharles Huchet, Paris, Union Générale d'éditions.

SAVES, Louis, 1984. "Cauterets »; "Saint-Sauveur » in Le thermalisme dans les HautesPyrénées. Catalogue d'exposition, Lourdes, Musée Pyrénéen.

SEBILLOT, Paul, 1968 Folklore de France, Paris, Maisonneuve et Larose, 4 vol. (1 $1^{\text {̀̀re }}$ éd. : 1904-1907)

TAMAROZZI, Federica, 1999. «La voce della sorgente. Parole di malattia e guarigione nella pratica termale in Corsica », Archivio Anthropologico Mediterraneo, anno II, n. 1 /2 : 41-69. 